\title{
Optic neuritis as an early sign of multiple sclerosis
}

This article was published in the following Dove Press journal:

Eye and Brain

26 October 2016

Number of times this article has been viewed

\section{Nilufer Kale \\ Department of Neurology, Bakirkoy Prof Dr Mazhar Osman Training and Research Hospital, Istanbul, Turkey}

Correspondence: Nilufer Kale Department of Neurology, Bakirkoy Prof Dr Mazhar Osman Training and Research Hospital, Istanbul, 33484 Turkey

Email kalenilufer@yahoo.com
Abstract: Optic neuritis $(\mathrm{ON})$ is an acute inflammatory demyelinating disorder of the optic nerve. The general characteristics of isolated $\mathrm{ON}$ include unilateral, subacute, and painful visual loss without systemic or other neurological symptoms. The etiology for ON varies including demyelinating disorders or infections, inflammation, toxic reasons, and genetic disorders. In most cases the responsible etiology may not be known for $\mathrm{ON}$ and in this case, it is termed idiopathic $\mathrm{ON}$. When a patient presents with an initial episode of ON, patients should undergo further tests. Assessing the patient with routine blood work, magnetic resonance imaging, cerebrospinal fluid tests, and visual evoked potentials provide further insight. In this review, we aimed to provide a review of $\mathrm{ON}$ as an initial symptom of multiple sclerosis and present clinical characteristics, therapy options, and recent literature.

Keywords: optic neuritis, multiple sclerosis, demyelination, corticosteroids, disease modifying treatments

\section{Introduction}

Optic neuritis $(\mathrm{ON})$ is an acute inflammatory demyelinating disorder of the optic nerve. The general characteristics of $\mathrm{ON}$ include unilateral, subacute, and painful visual loss without systemic or other neurological symptoms and ON is mostly seen in young females. ${ }^{1}$ The presentation is mostly monophasic but can also rarely be polyphasic with recurrent relapses. Atypical features include loss of pain prior to clinical presentation, optic pallor from the onset, complete vision loss with no improvement over the disease course, and a bilateral presentation. The etiology for $\mathrm{ON}$ varies including infections, inflammation, exposure to toxins, and genetic disorders. In most cases, the responsible etiology may not be known for ON, and in this case, it termed idiopathic ON. In some cases, ON can also be associated with demyelinating disorders of the central nervous system (CNS) including multiple sclerosis (MS) or neuromyelitis optica (NMO). Clinically isolated syndrome (CIS) is defined as a clinical demyelinating event that is isolated in time and mostly is the initial attack of MS. Patients with CIS may present with a wide variety of symptoms including sensory, pyramidal tract, brainstem symptoms, cerebellar involvement, ON, and transverse myelitis. CIS ON may be the initial presentation in $\sim 20 \%$ of MS patients, ${ }^{2-4}$ and $\mathrm{ON}$ presentation may occur during the course of the disease in $50 \%$ of patients with MS. ${ }^{5}$ Therefore, accurate diagnosis and risk assessment and management of patients with $\mathrm{ON}$ are warranted, and possibility of conversion to MS should be assessed. ${ }^{6}$

In this review, we aimed to provide a review of $\mathrm{ON}$ as an initial symptom of MS and present clinical characteristics, therapy options, and recent literature. 


\section{Characteristics of optic neuritis}

Common characteristic symptoms of ON include visual problems, periorbital pain, and color vision deficits. Vision loss is usually unilateral and deficits can range in severity from mild (20/20) to severe (no light perception). ${ }^{7,8}$ Patients usually complain about periocular, retro-ocular pain prior to vision loss that commonly occurs with eye movements (90\%). Visual field defects are also common in acute demyelinating ON. Different visual field patterns are seen in $\mathrm{ON}$ and include diffuse visual field defects or focal defects with centrocecal scotoma being the most common visual field defect. ${ }^{7,8}$ Altitudinal defects including visual field loss above or below the horizontal meridian are not usually expected and careful consideration of a differential diagnosis is warranted. ${ }^{9-14}$ Relative afferent pupillary defect (APD) is evaluated with the swinging flashlight test where each pupil is stimulated with a bright light in an alternating pattern, and reduced constriction of the affected and contralateral pupils in reaction to light is accepted as APD. The presence of APD would suggest unilateral optic neuropathy but it should be noted that in cases with prior ON in the fellow eye, APD would not be observed. ${ }^{5,7}$ Initial fundus exam is usually normal (retrobulbar $\mathrm{ON}$ ) while some patients might also present with mild disc edema (papillitis) (Figure 1). Vision defects usually progress for the first 1-2 weeks and then visual recovery usually begins within the first 4 weeks. ${ }^{7,8,9}$ Optic atrophy and disc pallor develops in 4-6 weeks. ${ }^{7,15,16}$ Additional findings in $\mathrm{ON}$ include abnormalities in low-contrast letter acuity, which is evaluated by the perception of light gray letters of progressively smaller size on a white background. Color vision is commonly affected and is evaluated by using various color plates (Ishihara color plates, American Optical Hardy-Rand-Rittler Color Vision Plates [HRR]). Contrast sensitivity is one of the affected parameters, which is evaluated as the minimum contrast level or shade of gray at which patients can perceive letters of a single large size. Contrast sensitivity seems to reflect disease progression and can be a

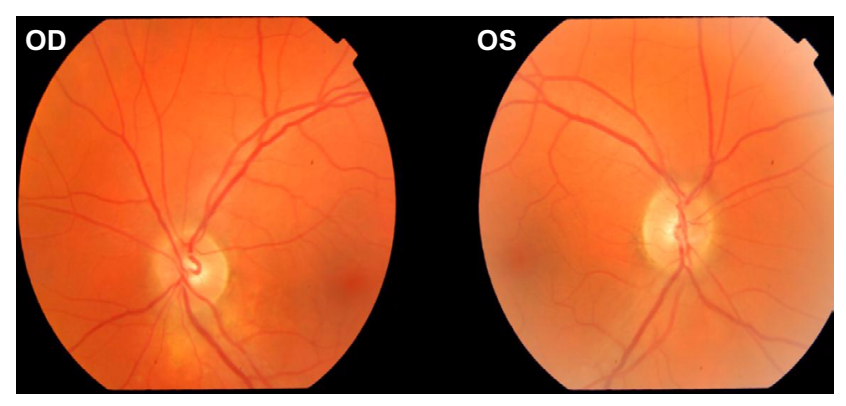

Figure I Fundus exam of a patient with $\mathrm{ON}$ on the right eye (OD). Abbreviations: OD, oculus dexter (right); OS, oculus sinister (left); ON, optic neuritis. valuable prognostic marker. These methods seem sensitive and help to detect visual dysfunction in patients with $\mathrm{ON}$. Recent studies have also demonstrated abnormalities in these modalities even in patients with normal vision $(\geq 20 / 20){ }^{5}$ Optic Neuritis Treatment Trial (ONTT) studied the visual function outcome of ON patients over 15 years and visual acuity, contrast sensitivity, and visual field parameters were evaluated. ${ }^{7,89}$ Among the patients with acute unilateral ON, $79 \%$ showed improvement by 3 weeks and $93 \%$ improved by 5 weeks. ${ }^{15,16}$ Rarely visual recovery was not complete and some patients failed to recover $(5 \%-10 \%) \cdot{ }^{7,8,15}$ However, it was suggested that long-term visual outcome was favorable for the majority of patients even when MS was present. ${ }^{9}$

\section{Differential diagnosis of optic neuritis}

When a patient presents with a demyelinating ON event with atypical features, the physician must take caution while assessing the patient. One of these atypical features include absence of pain prior to vision loss. However, painless vision loss may also be seen in $8 \%$ of the patients with typical ON. ${ }^{7}$ Severe visual loss with no light perception, progression of vision problems, or persistence of pain for $>2$ weeks, and lack of recovery even after a couple of weeks after the initial presentation are all unexpected features. ${ }^{7}$ Bilateral simultaneous or sequential $\mathrm{ON}$ is also not expected in common idiopathic ON but may be observed in NMO. Fundus exam also may show atypical findings, which include marked swelling of the optic nerve, retinal exudates, and peripapillary hemorrhages. Another unusual feature is bilateral ON, either simultaneously or sequentially, and NMO, NMO spectrum disorders should be considered. Patients with atypical ON features should be further evaluated for other possible diagnoses. Differential diagnosis include ischemic optic neuropathies, autoimmune disorders, rheumatologic and inflammatory conditions, compressive neuropathies, infections, and toxic, metabolic, or nutritional factors. ${ }^{9,17}$

\section{Diagnosis of optic neuritis and predicting conversion to multiple sclerosis}

$\mathrm{ON}$ is one of the most common initial clinical presentations of MS without any prior history of a demyelinating event. When a patient is referred to neurology with ON, after acute management, one of the most common questions would be the possibility of conversion to MS and thus longterm management. It is not always possible to predict the conversion or long-term prognosis of a clinical first event 
but some factors (sex, race, age, family history, neurological, and fundus findings) might be helpful to predict the risk of conversion to MS. Even though there is no a single biomarker to enable the neurologists to calculate this risk, magnetic resonance imaging (MRI) findings seem to predict strongly the risk of developing MS. ${ }^{17}$ When a patient presents with ON, neuroimaging should involve MRI of the brain and orbits (with fat saturation), spinal cord imaging would also provide of any additional MS lesions. Studies have shown that spinal cord lesions might be strong indicators for predicting MS risk. ${ }^{18}$ Usually the involved optic nerve would be hyperintense with contrast enhancement. If there are brain and spinal cord lesions compatible with MS, the characteristics of the demyelinating lesions would include $3 \mathrm{~mm}$ ovoid lesions that are mostly located in periventricular areas of the white matter and radiate toward the ventricular spaces. ${ }^{5}$ ONTT has also evaluated the risk of developing MS based on abnormal findings on initial MRI. While a patient with a first episode of ON who has normal brain MRI seems to have a lower risk of developing MS at 15 years (25\%), the chance of developing MS increases for patients with one or more lesions (72\%). ${ }^{4,16}$ Although it is not this review's aim to discuss radiologically isolated syndrome (RIS), it should be noted that since imaging has been utilized more in clinical practice, coincidental MRI findings compatible with MS are shown in asymptomatic patients with no neurological findings. Lebrun et al studied a group of patients with RIS and patients underwent paraclinical studies (blood, cerebrospinal fluid [CSF], and visual evoked potential [VEP] analysis) and follow-up with MRI. ${ }^{19}$ During the follow-up, a considerable proportion of the group developed CIS over a mean of 5.2 years $(33 \%)$. This study showed the valuable insight MRI would provide for diagnosis and predicting conversion to MS. ${ }^{19}$ In recent practice, McDonald criteria are the most utilized diagnostic criteria to assess the MS conversion risk in patients with a single demyelinating episode by providing MRI evidence for dissemination in space (DIS) and dissemination in time (DIT). In another study by Tintore et al, McDonald criteria were compared to Poser diagnostic criteria, in which the former seemed to be more sensitive (74\%), specific ( $86 \%$ ), and accurate for predicting conversion from CIS to clinically definite MS $(80 \%){ }^{20}$

While evaluating patients with a first episode of ON, in addition to MRI, additional tests including CSF analysis, evoked potentials (visual, motor, somatosensory, brain auditory) might also be helpful. CSF tests including routine parameters (cell count, glucose, protein levels, infection markers), oligoclonal banding (OB), and immunoglobulin
$\mathrm{G}(\mathrm{IgG})$ index seem to be useful for differential diagnosis of inflammatory, infectious disorders. While evaluating the CSF of patients with $\mathrm{ON}$, often the assessed parameters including protein and glucose levels should be within normal range, sometimes cell counts might be higher than normal. It has also been suggested that the presence of $\mathrm{OB}$ and IgG index might be a predictor of MS risk. ${ }^{16}$ However, in another study, Frederiksen et al suggested that the results of CSF parameters would not provide predictive values for developing clinically definite MS ${ }^{21}$ Yet, CSF studies would be recommended for differential diagnosis especially in atypical cases.

VEPs reflect demyelination in the afferent visual pathways. VEPs seem to be sensitive and specific for detecting ON even in silent cases with no apparent clinical presentation and abnormal findings are often observed in silent cases (65\%). ${ }^{22}$ Abnormal VEP findings include increased latencies and reduced amplitudes and abnormal waveforms. VEP might be useful to diagnose $\mathrm{ON}$; however, some studies have also reported that VEPs might not be useful in differentiating the cause of $\mathrm{ON}$ during the acute phase but may aid in subclinical cases with vision problems. ${ }^{22}$ Yet VEP evaluation provides valuable information in indeterminate cases and aid in diagnosis.

Optical coherence tomography (OCT) is a new tool to evaluate the thickness of retinal nerve tissues and retinal nerve fiber layers (RNFL). OCT has been reported as a noninvasive, safe, and easy technique that uses nearinfrared light to function. OCT studies have demonstrated the thinning of RNFL in patients with a prior history of ON, as well as in patients with MS. Recent evidence has shown that RNFL thickness reflects axonal degeneration and atrophy. OCT findings seem to be related to visual impairment as well as disease progression. ${ }^{23,24}$ Follow-up with OCT and assessing RNFL thickness may predict visual recovery after an ON relapse, and lower RNFL values may be correlated with impaired visual function. OCT might be a useful marker for axonal function of the optic nerves after ON. ${ }^{18}$

Recently studies utilizing B-mode transorbital ultrasonography for ON have also been reported. Application of transorbital sonography might reveal a thickening of the retrobulbar portion of the optic nerve during an ON relapse reflecting inflammation. Transorbital sonography seems to be a sensitive, highly accessible, and user-friendly technique. Further long-term studies are needed for the application of this method. ${ }^{25}$

\section{ON and NMO}

$\mathrm{NMO}$ is an inflammatory demyelinating disorder of the CNS affecting the optic nerves and spinal cord. Transverse myelitis 



Figure 2 The visual field defect of a patient with NMO, NMOlgG+.

Abbreviation: NMO, neuromyleitis optica.

in NMO is longitudinally extensive involving more than three vertebral segments. Vision loss presents with the clinical characteristics of ON but may be bilateral simultaneously or over time leading to atrophy and degeneration of nerve fiber layers (Figure 2). It may sometimes be challenging to distinguish NMO from MS; however, it is crucial to make an accurate diagnosis for monitoring and treating patients. Lennon et al showed an autoantibody against the astrocytic water channel aquaporin-4 (NMOIgG) that is highly sensitive and specific for NMO. ${ }^{26}$ There seems to be distinct MRI characteristic differences between NMO and MS-associated $\mathrm{ON}$ that helps in differentiating these two neuroinflammatory diseases at initial presentation. ${ }^{27}$ Mealy et al showed that NMO lesions were longitudinally extensive (measuring at least $17.6 \mathrm{~mm}$ in length) and would involve at least three optic nerve segments. ${ }^{27}$ Conversely, MS lesions were more commonly focal in one optic nerve segment localized anteriorly. This finding seemed to be specific ( $76.9 \%$ ) and sensitive $(80.8 \%)$ for NMO. OCT is also a useful method for showing axonal degeneration in optic nerve fibers and may help in distinguishing MS and NMO. Recent studies showed that ON in NMO typically would result in severe RNFL and ganglion cell layer thinning and microcystic macular edema leading to atrophy. Furthermore, silent optic nerve lesions and RNFL thinning may occur in MS but it is rare in NMO. In a study by Bichuetti et al, RNFL thickness in relapsing remitting MS, $\mathrm{NMO}$, and one of the atypical recurrent optic neuropathies named chronic relapsing inflammatory ON (CRION) were all compared. ${ }^{28}$ The frequency of ON for CRION seemed to be the highest (100\%), while $84 \%$ NMO patients and 34\% relapsing remitting MS patients presented with ON. When clinical features including visual acuity, prognosis, and RNFL thickness were all compared, NMO and CRION seemed to be doing worse but there were no differences between NMO and CRION. Evaluating RNFL thickness provides valuable information while differentiating MS, NMO, and CRION. Even though NMO and CRION are accepted as different disorders, there are similarities regarding these diseases, and further studies are needed to look for a possible shared mechanism. OCT seems to be a valuable test in differentiating NMO from MS and could be used as an outcome parameter and to monitor disease progression..$^{28,29}$

\section{Management of ON}

When a patient presents with vision problems and is diagnosed with ON, management strategies including acute and long-term therapies need to be made. Patients will also raise questions about the possibility of conversion to MS. To date, there is still no consensus about acute management strategies for $\mathrm{ON}$ as well as planning long-term treatments. 


\section{Acute management of $O N$}

Corticosteroids (CS) have been recommended for treating acute ON relapses. There is still no consensus about the application of CS (intravenous [iv] or oral forms), dosage, and treatment duration. Often, clinical presentation of optic neuritis is the main factor for the decision maker for initiating and duration of acute therapy. ONTT is a multicentered randomized clinical trial that assessed multiple parameters for patients with ON. ONTT also questioned acute management strategies and in a previous study, patients were assigned to three groups: 1) patients on oral prednisone $(1 \mathrm{mg} / \mathrm{kg} / \mathrm{d}$ for 14 days), 2) patients who received iv methylprednisolone (MP, $250 \mathrm{mg}$ every 6 hours for 3 days) followed by an oral prednisone taper ( $1 \mathrm{mg} / \mathrm{kg} / \mathrm{d}$ for 11 days), or 3) patients on oral placebo (for 14 days). Each regimen was followed by a short oral dosage taper of prednisone or placebo on days 15,16 , and 18. ONTT showed that while high-dose CS accelerated visual recovery, long-term visual outcome did not change when compared to the placebo. Visual outcomes for the patient group on oral CS did not improve and ONTT results even showed an increased recurrence rate in the same or fellow eye (twofold). Thus, the recommendation of ONTT is to treat acute ON with iv high-dose CS. Oral CS treatments did not seem to have a beneficial effect and thus they are not recommended. ${ }^{30,31}$ When patients were followed up for 2 years after initial treatment, the risk of MS conversion seemed to be lower in the high-dose CS group: $8 \%$ of the iv CS group, $18 \%$ of the placebo group, and $16 \%$ of the oral steroid group developed MS. ${ }^{3,31}$ The probability of developing MS between 5 and 10 years was low when patients had no lesions on MRI (7\%) but the risk increased when patients had $\geq 1$ lesions on MRI (27\%). ${ }^{7,15}$ ONTT recommended against oral prednisone alone in standard doses and treatment with iv methyprednisolone followed by oral prednisone or no treatment at all might be treatment options for acute ON cases. However, a recent review suggested that there was no conclusive evidence about the benefits of either intravenous or oral CS treatment regimens in terms of recovery to normal visual acuity, visual field, or contrast sensitivity. ${ }^{32}$ To date, many issues regarding the acute management of patients with isolated acute idiopathic ON still remain to be concluded.

\section{Plasma exchange}

In patients with severe optic function loss who are resistant to high-dose CS, plasma exchange (PE) is an alternative acute management strategy. Recent studies showed improvement of vision in patients with severe $\mathrm{ON}$ who failed to respond to high-dose CS therapy after PE. PE is a nonselective extracorporeal blood purification process with elimination of plasma and subsequent substitution. This also seems to be in accordance with the positive effects of PE in patients with other inflammatory demyelinating syndromes, including NMO who have been unresponsive to high-dose CS therapy. ${ }^{33,34,35}$ PE should be considered in resistant cases before optic atrophy develops. Immunoadsorption (IA) has been also offered as an alternative treatment to PE. IA is a selective technique for the removal of autoantibodies and immune complexes with less adverse effects. IA for the treatment of steroid-refractory relapse seems safe and effective, however, to date few studies have been reported on the treatment of MS by using IA. ${ }^{36}$

\section{Intravenous immunoglobulin}

Intravenous immunoglobulin (IVIG) is rarely used in ON patients and there is still conflicting data relating to the effects of IVIG. A recent study reviewed patients with severe visual loss unresponsive to high-dose CS who were treated with IVIG. The study showed a significant visual improvement in the IVIG group when compared to the controls. ${ }^{37}$ In another study, IVIG showed some benefit in patients with resolved ON and residual visual defects. ${ }^{15}$ However, some randomized trials failed to show improvement in visual acuity or contrast sensitivity after IVIG therapy. ${ }^{1}$ In atypical progressive ON cases where treatment options have failed or remain to be limited, IVIG might be considered.

\section{Long-term management of $\mathrm{ON}$}

After patients are treated for acute demyelinating ON, they should be followed up by a neurologist closely for MS conversion and therefore long-term treatment decisions. Immunomodulating therapies (DMTs) have been recommended for patients with a first demyelinating episode who have high MS conversion risk, and recent literature has suggested improved clinical outcomes and neuroimaging findings for this patient group when compared to the nontreated group. Studies showed a reduced MS conversion rate in high-risk patients with a CIS on DMTs. However, who and when to treat has long been debated and still remains inconclusive. Assessment of MRI after an initial ON episode provides insight for understanding the risk of converting to CDMS, the long-term risk for developing MS for patients with only symptomatic lesion and no additional MRI lesions was not high (20\%). It should also be noted that incomplete recovery from a demyelinating event could lead to permanent disability, and therefore, the main objective for long-term management would be to prevent further relapses. In addition 
to MRI, CSF analysis has also been assessed for diagnosis and monitoring disease course; however, CSF findings have also been debated. Prospective, population-based studies to estimate the conversion risk based on MRI findings and other laboratory tests are warranted. Even early in the disease course and after a single acute demyelinating ON episode, there is evidence of early neuronal degeneration and axonal damage and for patients with high risk of MS, DMTs should be considered. As previously stated, the presence of white matter lesions on the initial brain MRI was shown to be the strongest predictor for MS conversion in ONTT. The 15-year risk of developing MS was $25 \%$ in the patient group with no lesions, while the risk was $75 \%$ in the patient group with one or more lesions. ${ }^{38}$ Previously, various studies including the Controlled High Risk Avonex, Multiple Sclerosis Study, the Early Treatment of Multiple Sclerosis Study, the Betaseron in Newly Emerging Multiple Sclerosis for Initial Treatment Trial, the Prevention of Relapses and Disability by Interferon $\beta$-1a Subcutaneously in Multiple Sclerosis, and PreCISe trials have all helped to clarify the natural history of CIS including $\mathrm{ON}$ and management options for a single demyelinating episode. ${ }^{39-45}$ These studies suggested that early initiation of disease modification after a first clinical episode including treatment with interferons (IFN beta-1a and IFN beta 1-b) or glatiramer acetate would be beneficial in lowering the risk of conversion to MS as well as decreasing development of new T2 lesions on MRI. It is crucial to diagnose patients accurately and other possible etiologies including NMO, NMO spectrum disorders or vasculitis should be considered while evaluating the patients prior to initiating DMT.

\section{Conclusion}

The clinical presentation of MS and the disease course of MS are variable, including a single demyelinating event/ CIS, relapsing-remitting, primary-progressive, secondaryprogressive, or progressive-relapsing MS. Recently, a new term has been defined as RIS which has no clinical findings but coincidental a MRI reveals hyperintense lesions compatible with MS. To date, a single test for definite MS diagnosis is still not available and prognostic markers are still warranted to evaluate development of axonal degeneration or treatment effects of DMTs. Various authors have proposed different MS criteria based on DIS and DIT of CNS lesions and exclusion of other diseases. According to the Milo and Miller criteria, MS can be diagnosed clinically by demonstrating two separate attacks involving at least two different areas of the CNS. ${ }^{45}$ McDonald criteria have incorporated defined MRI criteria for DIS and DIT that provided guidance on how to diagnose MS after CIS. The most recent criteria are the revised $2010 \mathrm{McD}$ onald criteria that have simplified the MRI requirements for DIS and DIT so that an earlier diagnosis from a baseline brain MRI would be possible in cases that have both silent contrast-enhancing and non-enhancing hyperintense lesions. ${ }^{46,47}$ Despite all these efforts for accurate diagnosis, many questions for accurate management strategies after a single demyelinating episode remain to be solved. While ONTT has recommended high-dose iv CS for acute demyelinating $\mathrm{ON}$, there are conflicting studies as well. Additionally, there is still debate about when to initiate treatment as well as the dosage and duration of therapy. Chan et al suggested that when patients have hyperintense MRI lesions compatible with MS, the risk of developing MS following ON or other CIS increases. ${ }^{48}$ In the literature, prospective, randomized, double-blinded, placebo-controlled studies have suggested that the initiation of DMTs after CIS seemed to be beneficial for reducing MS conversion. ${ }^{39-45}$ It should also be noted that, while ONTT has shown that a significant percentage of $\mathrm{ON}$ patients who had an initial abnormal MRI had a second relapse in 15 years, $28 \%$ of patients with initial abnormal MRI remained relapse free for 15 years. All these findings demonstrate the challenge about predicting who will develop MS after a CIS or who will have a benign disease course. ${ }^{49}$ However, new McDonald and DIS criteria seem to be simpler and more sensitive and might provide accurate early MS diagnosis. ${ }^{46,50}$ Despite these important advances in the diagnosis of MS, questions still remain regarding the application and the implications of the new criteria in the daily practice and in clinical trials. Most importantly, thorough clinical evaluation and judgment along with careful differential diagnosis still remain the basis in the diagnosis of MS and caution is necessary when diagnosing a patient with MS. Long-term clinical studies have provided valuable information about the clinical features and treatment strategies for acute demyelinating $\mathrm{ON}$ as a clinical first event of MS. However, better diagnostic methods are still warranted to accurately predict MS conversion and the factors influencing disease severity to determine best appropriate therapeutic paradigm and avoid inaccurate treatments.

\section{Disclosure}

The author reports no conflicts of interest in this work.

\section{References}

1. Shams PN, Plant GT. Optic neuritis: a review. Int MS J. 2009;16(3): 82-89.

2. Abou Zeid N, Bhatti MT. Acute inflammatory demyelinating optic neuritis: evidence-based visual and neurological considerations. Neurologist. 2008;14(4):207-223. 
3. Beck RW, Gal RL. Treatment of acute optic neuritis: a summary of findings from the optic neuritis treatment trial. Arch Ophthalmol. 2008;126(7):994-995.

4. Brodsky M, Nazarian S, Orengo-Nania S, et al; Optic Neuritis Study Group. Multiple sclerosis risk after optic neuritis: final optic neuritis treatment trial follow-up. Arch Neurol. 2008;65:727-732.

5. Balcer LJ. Optic neuritis. N Engl J Med. 2006;354:1273-1280.

6. Avasarala JR. Clinically isolated syndrome-rethinking the diagnosis. J Neurol Sci. 2015;353(1-2):79-80.

7. Optic Neuritis Study Group. Visual function 15 years after optic neuritis: a final follow-up report from the optic neuritis treatment trial. Ophthalmology. 2008;115(6):1079-1082.

8. Beck RW, Cleary PA, Backlund JC; Optic Neuritis Study Group. The course of visual recovery after optic neuritis: experience of the optic neuritis treatment trial. Ophthalmology. 1994;101(11):1771-1778.

9. Beck RW, Cleary PA; Optic Neuritis Study Group. Recovery from severe visual loss in optic neuritis. Arch Ophthalmol. 1993;111(3):300.

10. Menon V, Saxena R, Misra R, Phuljhele S. Management of optic neuritis. Indian J Ophthalmol. 2011;59:117-122.

11. Keltner JL, Johnson CA, Cello KE, Dontchev M, Gal RL, Beck RW; Optic Neuritis Study Group. Visual field profile of optic neuritis: a final follow-up report from the optic neuritis treatment trial from baseline through 15 years. Arch Ophthalmol. 2010;128(3):330-337.

12. Keltner JL, Johnson CA, Spurr JO, Beck RW. Comparison of central and peripheral visual field properties in the optic neuritis treatment trial Am J Ophthalmol. 1999;128:543-553.

13. Fang JP, Donahue SP, Lin RH. Global visual field involvement in acute unilateral optic neuritis. Am J Ophthalmol. 1999;128:554-565.

14. Trobe JD, Beck RW, Moke PS, Cleary PA. Contrast sensitivity and other vision tests in the optic neuritis treatment trial. Am J Ophthalmol. 1996;121:547-553.

15. Balcer LJ, Baier ML, Cohen JA, et al. Contrast letter acuity as a visual component for the multiple sclerosis functional composite. Neurology 2003;61:1367-1373.

16. Dooley MC, Foroozan R. Optic neuritis. J Ophthalmic Vis Res. 2010;5(3):182-187.

17. Mamarabadi M, Razjouyan H, Mohammadi F, Moghaddasi M. Assessment of outcome predictors after first attack of optic neuritis. Can J Neurol Sci. 2011;38:887-895.

18. Swanton JK, Fernando KT, Dalton CM, et al. Early MRI in optic neuritis: the risk for disability. Neurology. 2009;72(6):542-550.

19. Lebrun C, Bensa C, Debouverie M, et al. Association between clinical conversion to multiple sclerosis in radiologically isolated syndrome and magnetic resonance imaging, cerebrospinal fluid, and visual evoked potential: follow-up of 70 patients. Arch Neurol. 2009;66(7):841-846.

20. Tintoré M, Rovira A, Río J, et al. New diagnostic criteria for multiple sclerosis: application in first demyelinating episode. Neurology. 2003;60(1):27-30.

21. Frederiksen JL, Larsson HB, Olesen J. Correlation of magnetic resonance imaging and CSF findings in patients with acute monosymptomatic optic neuritis. Acta Neurol Scand. 1992;86(3):317-322.

22. Chan WJ. Recent advances in optic neuritis related to multiple sclerosis. Acta Ophthalmol. 2011;90(3):203-209.

23. Frohman E, Costello F, Zivadinov R, et al. Optical coherence tomography in multiple sclerosis. Lancet Neurol. 2006;5(10):853-863.

24. Costello F, Coupland S, Hodge W, et al. Quantifying axonal loss after optic neuritis with optical coherence tomography. Ann Neurol. 2006;59(6):963-969.

25. Lochner P, Leone MA, Coppo L, et al. B-mode transorbital ultrasononography for the diagnosis of acute optic neuritis. A systematic review. Clin Neurophysiol. 2016;127(1):803-809.

26. Lennon VA, Wingerchuk DM, Kryzer TJ, et al. A serum autoantibody marker of neuromyelitis optica: distinction from multiple sclerosis. Lancet. 2004;364(9451):2106-2112.

27. Mealy MA, Whetstone A, Orman G, Izbudak I, Calabresi PA, Levy M. Longitudinally extensive optic neuritis as an MRI biomarker distinguishes neuromyelitis optica from multiple sclerosis. J Neurol Sci. 2015;15;355(1-2):59-63.
28. Bichuetti DB, de Camargo AS, Falcão AB, Gonçalves FF, Tavares IM, de Oliveira EM. The retinal nerve fiber layer of patients with neuromyelitis optica and chronic relapsing optic neuritis is more severely damaged than patients with multiple sclerosis. J Neuroophthalmol. 2013;33(3):220-224.

29. Bennett JL, de Seze J, Lana-Peixoto M, et al. Neuromyelitis optica and multiple sclerosis: seeing differences through optical coherence tomography. Mult Scler. 2015;21(6):678-688.

30. Optic Neuritis Study Group. The 5-year risk of MS after optic neuritis: experience of the optic neuritis treatment trial. Neurology. 1997;49(5):1404-1413.

31. Gal RL, Vedula SS, Beck R. Corticosteroids for treating optic neuritis. Cochrane Database Syst Rev. 2012;8:CD001430.

32. Ruprecht K, Klinker E, Dintelmann T, Rieckmann P, Gold R. Plasma exchange for severe optic neuritis treatment of 10 patients. Neurology. 2004;63:1081-1083.

33. Keegan M, Pineda AA, McClelland RL, Darby CH, Rodriguez M, Weinshenker BG. Plasma exchange for severe attacks of CNS demyelination:predictors of response. Neurology. 2002;58:143-146.

34. Roesner S, Appel R, Gbadamosi J, Martin R, Heesen C. Treatment of steroid-unresponsive optic neuritis with plasma exchange. Acta Neurol Scand. 2012;126(2):103-108.

35. Heigl F, Hettich R, Arendt R, Durner J, Koehler J, Mauch E. Immunoadsorption in steroid-refractory multiple sclerosis: clinical experience in 60 patients. Atheroscler Suppl. 2013;14(1):167-173.

36. Tselis A, Perumal J, Caon C, et al. Treatment of corticosteroid refractory optic neuritis in multiple sclerosis patients with intravenousimmunoglobulin. Eur J Neurol. 2008;15(11):1163-1167.

37. Roed HG, Langkilde A, Sellebjerg F, et al. A double-blind, randomized trial of IV immunoglobulin treatment in acute optic neuritis. Neurology. 2005;64(5):804-810.

38. Atkins EJ, Drews-Botsch CD, Newman NJ, Calvetti O,Swanson S, Biousse V. Management of optic neuritis in Canada: survey of ophthalmologists and neurologists. Can J Neurol Sci. 2008;35(2):179-184.

39. Jacobs LD, Beck RW, Simon JH, et al. Intramuscular interferon beta-1a therapy initiated during a first demyelinating event in multiple sclerosis. CHAMPS Study Group. N Engl J Med. 2000;343:898-904.

40. O'Connor P, Kinkel RP, Kremenchutzky M. Efficacy of intramuscular interferon beta-1a in patients with clinically isolated syndrome: analysis of subgroups based on new risk criteria. Mult Scler 2009;15:728-734.

41. The PRISMS (Prevention of Relapses and Disability by Interferon- $\beta$ 1a Subcutaneously in Multiple Sclerosis Study) Group and University of British Columbia MS $\backslash$ MRI Analysis Goup. PRISMS-4: long term efficacy of interferon beta-1a in relapsing MS. Neurology. 2001;56:1628-1636.

42. Kappos L, Polman CH, Freedman MS, et al. Treatment with interferon beta-1b delays conversion to clinically definite and McDonald MS in patients with clinically isolated syndromes. Neurology. 2006;67: 1242-1249.

43. Comi G, Martinelli V, Rodegher M, et al; PreCISe study group. Effect of glatiramer acetate on conversion to clinically definite multiple sclerosis in patients with clinically isolated syndrome (PreCISe study): a randomised, double-blind, placebo-controlled trial. Lancet. 2009;374(9700):1503-1511.

44. Comi G, Filippi M, Barkhof F, et al. Effect of early interferon treatment on conversion to definite multiple sclerosis: a randomised study (ETOMS). Lancet. 2001;357:1576-1582.

45. Milo R, Miller A. Revised diagnostic criteria of multiple sclerosis. Autoimmun Rev. 2014;13(4-5):518-524.

46. Gómez-Moreno M, Díaz-Sánchez M, Ramos-González A. Application of the 2010 McDonald criteria for the diagnosis of multiple sclerosis in a Spanish cohort of patients with clinically isolated syndromes. Mult Scler. 2012;18(1):39-44.

47. Tintoré M, Rovira A, Río J, et al. Baseline MRI predicts future attacks and disability in clinically isolated syndromes. Neurology. 2006;67:968-972.

48. Chan JW. Early diagnosis, monitoring, and treatment of optic neuritis. Neurologist. 2012;18(1):23-31.

49. Kale N. Management of optic neuritis as a clinically first event of multiple sclerosis. Curr Opin Ophthalmol. 2012;23(6):472-476. 


\section{Publish your work in this journal}

Eye and Brain is an international, peer-reviewed, open access journal focusing on clinical and experimental research in the field of neuroophthalmology. All aspects of patient care are addressed within the journal as well as basic research. Papers covering original research, basic science, clinical and epidemiological studies, reviews and evaluations,

Submit your manuscript here: https://www.dovepress.com/eye-and-brain-journal guidelines, expert opinion and commentary, case reports and extended reports are welcome. The manuscript management system is completely online and includes a very quick and fair peer-review system, which is all easy to use. Visit http://www.dovepress.com/testimonials.php to read real quotes from published authors. 